Tropical Journal of Pharmaceutical Research October 2012; 11 (5): 855-866

(C) Pharmacotherapy Group, Faculty of Pharmacy, University of Benin

Benin City, 300001 Nigeria.

All rights reserved.

Available online at http://www.tjpr.org

Review Article

http://dx.doi.org/10.4314/tjpr.v11i5.20

\title{
Poly(ethylene oxide)-Poly(propylene oxide)-Based Copolymers for Transdermal Drug Delivery: An Overview
}

\section{Evren Algin Yapar $^{1 *}$ and Özge İnal ${ }^{2}$}

${ }^{1}$ Ministry of Health of Turkey, Turkish Medicines and Medical Devices Agency, Söğütözü Mah. 2176. Sok. No:5 Kat:6, 06520 Çankaya-Ankara, '2Department of Pharmaceutical Technology, Faculty of Pharmacy, University of Ankara, 06100 Tandoğan- Ankara, Turkey

\begin{abstract}
Amphiphilic poly(ethylene oxide)-poly(propylene oxide) (PEO-PPO)-based copolymers are thermoresponsive materials having aggregation properties in aqueous medium. As hydrosolubilizers of poorly water-soluble drugs and improved stability of sensitive agents, these materials have been investigated for improvement in solubility, stability, release and bioavailability of drugs. The goal of the present review is to summarize the properties of PEO-PPO based copolymers and highlight the works that have been carried out on these materials for transdermal drug delivery.
\end{abstract}

Keywords: PEO-PPO based copolymer, Poloxamer, Hydrogel, Transdermal, Drug delivery. 


\section{INTRODUCTION}

Advances in pharmaceutical technology led to the design of technologically optimal vehicles for the administration of drugs, thus allowing improvement in the properties of the preparations as well as maximization of their stability, bioavailability and biocompatibility. However, a persisting drawback is low solubility in physiological aqueous environment of about $50 \%$ of approved active molecules, resulting in poor bioavailability [1]. Limited solubility also constitutes a hurdle in the development of topical formulations, and improved solubility usually correlates well with higher bioavailability [1-3].

Inclusion of hydrophobic molecules within polymeric micelles is a solution approach to enhanced drug solubilization, stability and bioavailability. Polymeric micelles are nanoscopic (> $100 \mathrm{~nm}$ ) structures formed by amphiphilic block copolymers composed of hydrophilic and hydrophobic chains that selfassemble in water, above a certain concentration named the critical micelle concentration (CMC) $[1,4]$. Micelles comprise of an inner and outer domain referred to as core and shell, respectively. Due to the hydrophobic nature of the core, these entities particularly suitable for the solubilization of water insoluble molecules, protection of unstable agents from chemical degradation and metabolism by biological agents, and sustained release in different formulations $[1,5]$. Among the polymers displaying micelleformation ability, the most broadly investigated amphiphilic materials are derivatives of PEO-PPO block copolymers $[1,6,7]$ which form swellable hydrogels. Swollen hydrogels, which give more emollient effect than conventional ointments due to their high water content, have been preferred for topical-transdermal drug delivery in recent years.

Transdermal delivery of drugs using PEOPPO-based polymeric micelles has been investigated for the delivery of mostly anti- inflammatory drugs [8-19], analgesic [20], local anesthetic [21], other drugs [19,22-24] and proteins $[25,26]$. In this review, we present an overview of the properties of PEO-PPO based copolymers and their applications in transdermal drug delivery.

\section{PROPERTIES OF PEO-PPO BASED COPOLYMERS}

This group of copolymers consists of ethylene oxide (EO) and propylene oxide (PO) blocks arranged in a triblock structure $E O_{x}-P_{y}-E_{x}$. Their chemical formula is $\mathrm{HO}[\mathrm{CH} 2-\mathrm{CH} 2 \mathrm{O}]_{x} \quad[\mathrm{CH}(\mathrm{CH} 3)-\mathrm{CH} 2 \mathrm{O}]_{y}[\mathrm{CH} 2-$ $\mathrm{CH} 2 \mathrm{O}]_{x} \mathrm{OH}$, where $\mathrm{y}$ is greater than 14 . Registered trademarks of these copolymers (e.g., Pluronic ${ }^{\circledR}$ Synperonic $^{\circledR}$ or Tetronic $^{\circledR}$ ) cover a large range of liquids, pastes and solids. Two families are available: (1) the linear and bifunctional PEO-PPO-PEO triblocks or poloxamers (Pluronic and Synperonic) and (2) the branched 4-arm counterparts named poloxamine (Tetronic) [1].

They are synthesized by sequential polymerisation of $\mathrm{PO}$ and $\mathrm{EO}$ monomers in the presence of sodium hydroxide or potassium hydroxide $[27,28]$. The initial stage of the synthesis includes growth of the PO block followed by the growth of EO chains at both ends of the PO block. Anionic polymerization usually produces polymers with a low polydispersity index $\left(M_{n} / M_{w}\right)$. However, the commercially available Pluronic preparations may contain admixtures of the PO homopolymer as well as di- and triblock copolymers, exhibiting lower degrees of polymerization than expected [29]. These copolymers are available in a wide range of molecular weights and EO/PO ratios [1]. They have amphiphilic properties characterised by their HLB values (hydrophilic-lipophilic balance), in which the number of hydrophilic EO $(x)$ and hydrophobic $\mathrm{PO}(y)$ units can be altered to vary the size, hydrophilicity and lipophilicity easily $[27,29]$. 
The most extensive work was performed on the poloxamer (Pluronic) group. The poloxamers consist of more than 30 different non-ionic surface-active agents. The poloxamer series covers a range of liquids, pastes, and solids, with molecular weights and EO-PO weight ratios varying from 1100 to 14,000 and $1: 9$ to $8: 2$, respectively. Concentrated aqueous solutions of poloxamer form thermoreversible gels [30].

A defining property of poloxamer is the ability of the individual block copolymer molecules, termed 'unimers', to self-assemble into micelles in aqueous solutions. These 'unimers' form a molecular dispersion in water at block copolymer concentrations below the critical micelle concentration (CMC). At concentrations of the block copolymer above the $\mathrm{CMC}$, the unimer molecules aggregate, forming micelles through a process called 'micellization'. The driving force for the micellization is the hydrophobic interactions of the PO blocks. The number of block copolymer unimers forming one micelle is referred to as the 'aggregation number'. Usually this number ranges from several to over a hundred. The process of transfer of water-insoluble compounds into the PO core of the micellar solution is referred to as 'solubilization' [29]. The gelation mechanism of poloxamer solutions has been investigated extensively, but is still being debated. Ultrasonic velocity, light-scattering and small-angle neutron scattering measurements of aqueous poloxamer solutions have clearly indicated a micellar mode of association [30,31-36]. Micelle formation occurs at the critical micellization temperature as a result of PPO block dehydration $[30,33,37]$. With increasing temperature, micellization becomes more important, and at a definite point, micelles come into contact and no longer move. Thus, packing of micelles and micelle entanglements may be possible mechanisms of poloxamer solution gelation with increased of temperature [30,38]. In addition, the formation of highly ordered structures, such as cubic crystalline phase, has been proposed as the driving force for gel formation [30,34-36,39], but this hypothesis has been questioned by some of the researchers [30]. In order to attain relatively stable gels, these applications require polymer concentrations of usually around 15 $w t \%[1,40-43]$.

Poloxamers (Pluronics) are nonionic surfactants possessing excellent wetting, antifoaming and solubilizing properties. Though their potential drawbacks include weak mechanical strength and rapid erosion (i.e., dissolution from the surface) [44,45], poloxamer gels have been shown to provoke neither skin irritation nor sensitivity, thus confirming its good tolerability and useful in topical formulations $[1,8,44,46]$. Among these, Poloxamer 407, also known as Lutrol or Pluronic F127, is an ABA-type triblock copolymer consisting of PEO units $(A=70 \%)$ and PPO units $(B=30 \%)$, which is transformed from a low-viscosity solution to a semisolid gel upon heating to body temperature at concentrations of $>20 \%$ $[47,48]$, have become the subject of growing interest for use in the formulation of dosage forms owing to their low toxicity and ability to form a clear solution or gel in aqueous media $[8,49]$. To increase their applicability, poloxamers can be physically or chemically modified by different polymenrs $[47,50,51]$.

\section{PEO-PPO BASED COPOLYMERS IN TRANSDERMAL DRUG DELIVERY}

Studies on PEO-PPO-based polymeric micelles for transdermal drug delivery have been widely carried out with Poloxamer 407 (Pluronic F127) and these studies mostly focused on small drug molecules in order of anti-inflammatory [8-19], analgesic [20], local anesthetic [21] and cardiac effectiveness [19,22-24] and rarely focused on big molecules such as arginine vasopressin (AVP) and insulin [25,26].

Trop J Pharm Res, October2012;11 (5): 857 


\section{Delivery of small molecules}

An interesting study based on a dual purpose gel was designed by Cafaggi et al to increase the aqueous solubility of tolfenamic acid, an anti-inflammatory drug, at least 2000 -fold at $25{ }^{\circ} \mathrm{C}$ through the use of Poloxamer 407 and evaluated a gel based on this poloxamer for the topical or transdermal administration of the drug [9]. The sustained release of tolfenamic acid towards the receptor phase constituted by isopropyl myristate was accompanied, in its early stage, by the concomitant release of ethanol and tetrahydrofurfuryl alcohol used as cosolvents to obtain a drug loading of $0.6 \%(\mathrm{w} / \mathrm{w})$. The poloxamer micellar phase was directly involved in the late stage of drug release, thus indicating that a strong interaction occurred in the gel between the poloxamer and tolfenamic acid. The results indicate the possibility of both systemic and topical administration of tolfenamic acid by means of aqueous solutions or gels containing Poloxamer 407 in an adequate concentration. The solubilization process was spontaneous and exothermic, as indicated by thermodynamic parameters [8].

The solubility of naproxen, another antiinflammatory agent, at $\mathrm{pH} 2$ was significantly increased as a linear function of Poloxamer 407 concentration and the micellar solubilization was spontaneous and exothermic. The release of naproxen from Poloxamer $407 \mathrm{gel}$ across the membrane into isopropyl myristate was dependent on medium $\mathrm{pH}$ and was significantly sustained at $\mathrm{pH} \mathrm{2,} \mathrm{with} \mathrm{an} \mathrm{inversely} \mathrm{proportional} \mathrm{to} \mathrm{the}$ surfactant concentration [10].

Various hydrogel formulations loaded with poorly water-soluble piroxicam for transdermal delivery has been investigated by some researchers $[11,12,15]$. The pharmacokinetics and anti-inflammatory activity of piroxicam from Poloxamer 407 gel were determined to investigate percutaneous absorption of piroxicam from poloxamer gels in rats; Poloxamer 407 gel containing $1 \%$ piroxicam showed significant inhibition of carragenin-induced rat foot swelling (\%) with a linear relationship of piroxicam dose within $\sim 0.4-3.2 \mathrm{mg} / \mathrm{kg}$ when compared to the control group (Figure 1).

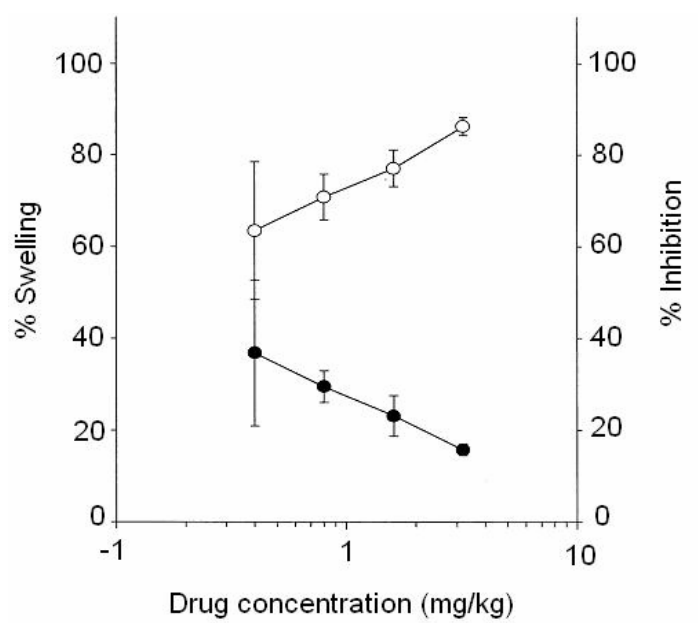

Figure 1: Dose response of piroxicam after paw injection into the sole of rat carrageenan-induced edema model; $0 \%$ swelling, $\bigcirc=\%$ inhibition [11].

Piroxicam diffusion in Poloxamer 407 hydrogel was evaluated with a new technique (UV imaging) and monitored by measuring the absorbance of light passing through the diffusion cell at $26{ }^{\circ} \mathrm{C}$, thus providing realtime concentration maps ( $7 \times 3 \mathrm{~mm}$ imaging area) within the gel as a function of time. This study indicates that UV imaging has great potential for measuring drug diffusion in and release from gel matrices. Compared to the currently used conventional techniques, this technology has several advantages including high information content, non-intrusive measurements without the need for labeling, flexibility with respect to experimental design and simplicity of operation [12].

The percutaneous absorption of a new synthetic capsaicin analog, a potent antiinflammatory analgesic drug, from a Poloxamer 407-based formulation was studied by Lee et al [18]. The results showed that the flux of poloxamer-based gels increased linearly as drug concentration 
increased and at $32{ }^{\circ} \mathrm{C}$ was higher for free base form of drug than that of its salt form. There was a variation in percutaneous absorption of the drug, depending on the species used and was highest in hairless mice but lowest in hamsters, and there was no skin toxicity. The poloxamer-based formulation appears useful in the systemic delivery of capsaicin as topical or transdermal patch formulations [18].

The feasibility of PEO-PPO-PEO copolymer with average molecular weight of $8400 \mathrm{gel}$ as a release vehicle for percutaneous administration of analgesic fentanyl was investigated by in vitro and in vivo studies. At a concentration of $46 \%$ at $37^{\circ} \mathrm{C}$, PEO-PPOPEO copolymers formed a gel and showed a pseudo-zero-order sustained-release profile as presented in Figure 2. Assessment of the effect of the copolymer on nude mouse skin showed a decrease in the apparent permeability coefficient and pharmacokinetics of the fentanyl patch was shown to be in steady state within $24 \mathrm{~h}$, and this was maintained for at least $72 \mathrm{~h}$. Thus, it appears that fentanyl preparations based on PEOPPO-PEO copolymer gel might be practical for percutaneous delivery [20].

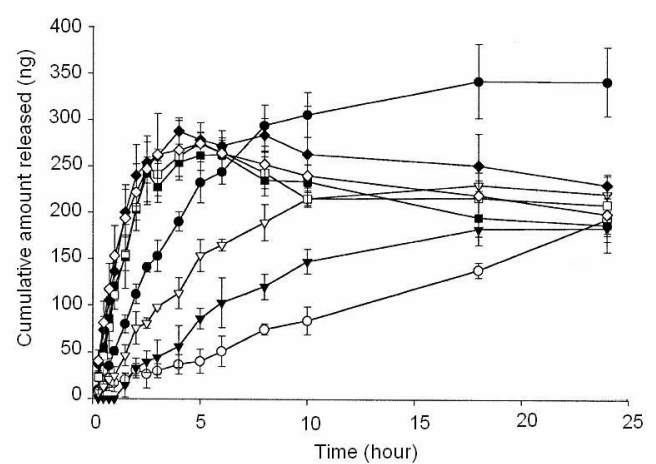

Figure 2: Effect of PEO-PPO-PEO copolymer concentration on fentanyl release from a cellulose membrane $\left(\bullet: \mathrm{H}_{2} \mathrm{O}, \mathrm{O}: 46 \%, \mathbf{\nabla}: 20 \%, \nabla: 10 \%\right.$, 口: $1 \%, \square: 0.1 \%, \diamond: 0.01 \%, \diamond: 0.001 \%$; each value represents the mean \pm S.D. of $n 53$ ) [20]

The percutaneous delivery of pranoprofen from Poloxamer 407 and hydroxypropyl methylcellulose (HPMC) gel mixture was investigated and it was found that the rate of drug permeation increased significantly with increasing drug concentration in the gels, up to $0.16 \%$, and increased with increasing temperature, with an activation energy (Ea) of $8.88 \mathrm{kcal} / \mathrm{mol}$ which highlighted the feasibility of a topical gel formulation of pranoprofen [13]. To develop lidocaine gels of enhanced local anesthetic effects, HPMC-Poloxamer 407 gels were formulated; as drug concentration in the gels increased up to $3 \%$, drug permeation rate linearly increased, reaching a plateau. Also, as the temperature of the surrounding solution increased, the permeation of drug increased as well [21].

Suedee et al [23] aimed to develop a transdermal patch for selective controlled delivery of the active $S$ enantiomer of racemic propranolol, and to evaluate its performance in vivo using Wistar rats. A molecularly imprinted polymer (MIP) thin-layer composited cellulose membrane having selectivity for S-propranolol was employed as enantioselective-controlled release system. The effect of gel reservoir (poloxamer and chitosan) on enantioselective drug delivery was investigated. The chitosan gel allowed excellent selectivity for delivery of the Spropranolol enantiomer, whilst the more rheologically structured poloxamer gel formulation provided no selective release of S-propranolol. The chitosan gel exhibited high flux and had the ability to enantioselective deliver S-propranolol across excised rat skin. The results demonstrated that transdermal patch based on the MIP composite membrane-controlled release system may have potential in enantioselective-controlled delivery of Sisomer of racemic propranolol [23].

Transdermal delivery of timolol (TM) from a Poloxamer 407 gel reservoir system has been investigated; it was found that both the gel concentration and the artificial membrane can be used as "tools" to regulate TM delivery from gel reservoir. At low concentrations of Poloxamer 407 and high pore size of the membranes (Polyflux $®$, 
PES-30), the stratum corneum mainly controls TM delivery. At high Poloxamer 407 concentrations and low pore size of membranes (NF-PES-10), the contribution of the device (gel+artificial membrane) on the delivery of TM was found to be significant [24].

\section{Effect of additives on the delivery of small molecules from poloxamers}

Among the enhancers, non-ionic surfactants have attracted attention in drug delivery from poloxamers-based systems. The enhancing effect of polyoxyethylene-2-oleyl ether, a nonionic surfactant, on percutaneous absorption of piroxicam from Poloxamer 407 gel was evaluated in rats by Shin et al [11]. The piroxicam gel containing polyoxyethylene-2oleyl ether increased the relative bioavailability $\sim 1.8$-fold compared with the gel without enhancer. Percutaneous administration of piroxicam gel containing polyoxyethylene-2-oleyl ether to rats showed relatively constant, sustained blood concentration with minimal fluctuation [11].

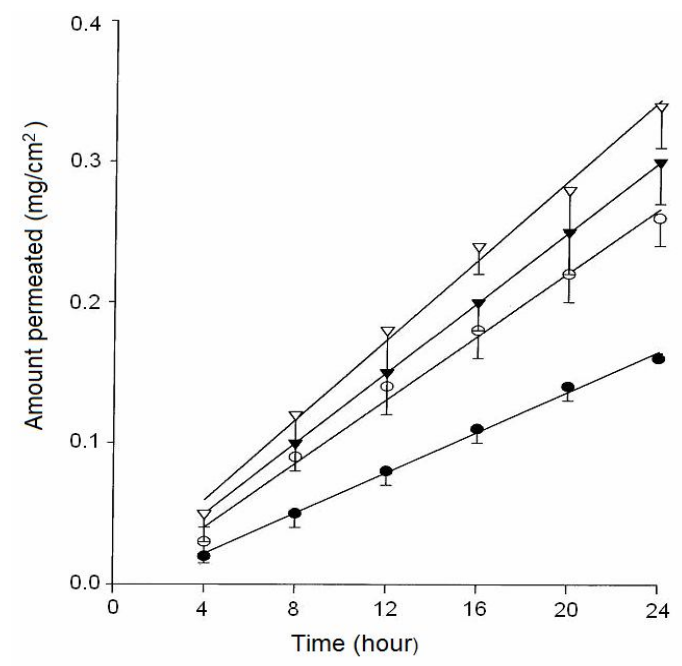

Figure 3: Effect of non-ionic surfactants in the poloxamer gel containing $1 \%$ piroxicam on drug permeation through excised rat skin $(0=$ control, $\bigcirc=$ polyoxyethylene-23-laurylether, $\boldsymbol{\nabla}=$ polyoxyethylene-2-oleylether, $\nabla=$ polyoxyethyl-ene-2stearyl ether) [15].
The enhancing effect of non-ionic surfactants on the permeation of piroxicam from the poloxamer gels was evaluated in another study by Shin et al [15]. The effectiveness of penetration enhancers, measured by ratio of piroxicam flux in the presence or absence of enhancers (enhancement factor) indicate that polyoxyethylene-2-oleyl ether showed the highest enhancing effects (Figure 3 ) with an enhancement factor of 2.84 among the studied surfactants. Thermal analysis revealed that various surfactants have different fluidizing effects on stratum corneum, and skin pretreated with the Poloxamer 407 gels containing various surfactants showed a loosely layered stratum corneum and wide intercellular space that elucidated the mechanisms of the action of enhancers [15].

The effect of four terpene enhancers, enhancer lipophilicity and ethanol concentration using hydroxypropyl cellulose (HPC) and two Poloxamer 407 gel formulations on the in vitro percutaneous permeation of ketoprofen from hairless mouse skin were evaluated by El-Kattan et al [17]. The highest increase in ketoprofen permeation was observed using limonene followed by nerolidol, fenchone and thymol for all formulations. Moreover, ethanol had a synergistic effect on the enhancing activity of the terpenes [17]. In another study, effect of two terpenes ( $d$-limonene and 1-menthol) were evaluated for $20 \%$ ibuprofen in $30 \%$ Poloxamer 407 formulation. Avoiding gel formation and in order to assess their drug diffusion/penetration (permeation) into the skin, it was revealed that the superior enhanced permeationof terpenes, especially $d$-limonene, was dependent on ibuprofen in fluid preparations which may be useful as a topical preparation of ibuprofen [14].

The penetration of sodium naproxen, formulated in Poloxamer 407 gels containing Azone and Transcutol as penetration enhancers, was studied through human skin in vivo. It was found that the combination of Azone and Transcutol in Pluronic F127 gels 
enhanced sodium naproxen penetration, with enhancement ratios of up to two-fold compared with the formulation containing only Transcutol. This was confirmed by transepidermal water loss and attattentuated total reflectance FTIR spectroscopy analyses by Escobar-Chavez et al [16].

The effect of different types of enhancers was evaluated on the permeation rate of lidocain through skin from HPMC-Poloxamer 407 gels. Among the enhancers, such as glycols, non-ionic surfactants and bile salts, diethylene glycol showed the greatest enhancing effect on drug permeation through skin, and in the area under the efficacy curve (AUEC) of the rat-tail flick tests, lidocaine gel containing diethylene glycol showed about 3.89-fold increase in analgesic activity compared with control [21].

The effect of dimethyl sulphoxide (DMSO) on the micellization, gelation and dissolution properties of aqueous poloxamer solutions, clearly showed that DMSO induces reductions in critical micellization and gelation temperatures of poloxamer systems analyzed by differential scanning calorimetry and tube inversion experiments. In addition, high resolution solid state $1 \mathrm{H}$ magic angle spinning nuclear magnetic resonance analyses provided indications of the specific chemical groups in the poloxamer affected by DMSO, and the molecular mechanism involved. The presence of DMSO accelerated dissolution of the pure gel in water and the release of a hydrophobic drug (flufenamic acid) from poloxamer gel, while it reduced the release of a hydrophilic drug (metoprolol tartrate) [19].

The crystallization of drug in a matrix may significantly affect the efficacy and quality of a transdermal drug delivery system. Therefore, control of drug crystallization is of particular interest in the development of efficient transdermal delivery systems. From this point of view, the crystallization inhibition effect of poloxamer was investigated as an additive and it was found that poloxamer significantly inhibited the crystallization of ketoprofen in a polyisobutylene adhesive matrix, in a study by Kim and Choi [52].

The salts commonly included in the formulation of Poloxamer 407 gels can dramatically change the release rate of a drug. In a salt effect study, the release rate and diffusion coefficient of propranolol, a model drug, were significantly reduced when $\mathrm{NaCl}, \mathrm{Na}_{2} \mathrm{SO}_{4}, \mathrm{NaH}_{2} \mathrm{PO}_{4}, \mathrm{MgSO}_{4}$ and $\mathrm{CaCl}_{2}$ were added to the gels of $21 \%$ Poloxamer 407 in an aqueous medium at $37{ }^{\circ} \mathrm{C}$ through a cellulose membrane, as presented in Figure 4. The magnitude of the effect on release rate depends on the nature and concentration of the salt. Since propranolol is expected to be located outside the micelles in the gel, these salt effects have been attributed to increase in the microviscosity of the aqueous regions of the gel, rather than to a change in micellization behavior. It is analogous to the change in the aggregation and phase behavior of aqueous solutions of non-ionic surfactants in the presence of salts [22].

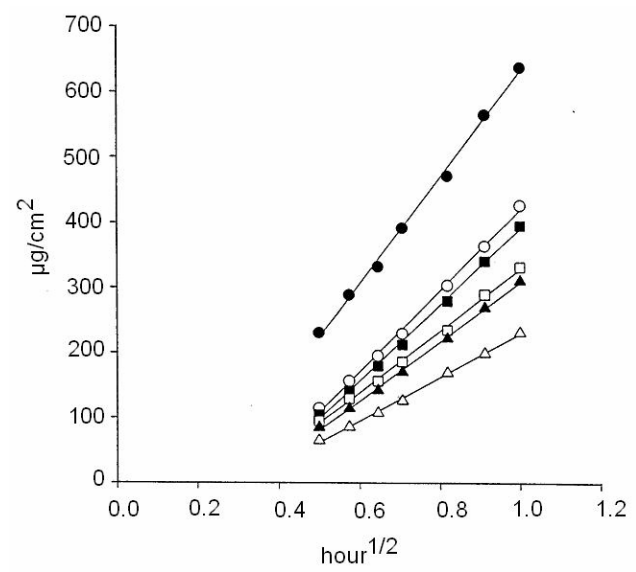

Figure 4: Effects of 0.1 molal concentrations of various salts on release rate of propranolol from a 21\% Poloxamer 407 gel at $37^{\circ} \mathrm{C}$. (O: no salt, $\bigcirc$ : $\mathrm{CaCl}_{2}$, $\boldsymbol{\square}: \mathrm{NaCl}, \square: \mathrm{MgSO}^{4}, \boldsymbol{\Delta}: \mathrm{NaH}_{2} \mathrm{PO}_{4}, \triangle$ : $\mathrm{Na}_{2} \mathrm{SO}_{4}$; Linear regression lines are fitted to the data) [22].

The influence of the addition of lecithin, a permeation enhancer, on the rheological and in vitro permeation properties of Poloxamer 
407 gels was investigated by Vito'ria et al [53].

Light microscopy and rheological parameters were used to characterize the microscopic structure of the formulation that showed nonNewtonian behaviour, i.e., pseudoplastic flow with a yield value (Figure 5). The results revealed that increased concentration of lecithin increased the thixotropy, yield value, apparent viscosity, and gelation temperature of the gels. Light microscopy showed the formation of micellar structures following the addition of lecithin, which may account for changes in rheological properties. In vitro permeation of a model drug, triamcinolone acetonide, was decreased when lecithin concentration was increased. The presence of lecithin in the poloxamer gel improved the characteristics for topical drug delivery [53].

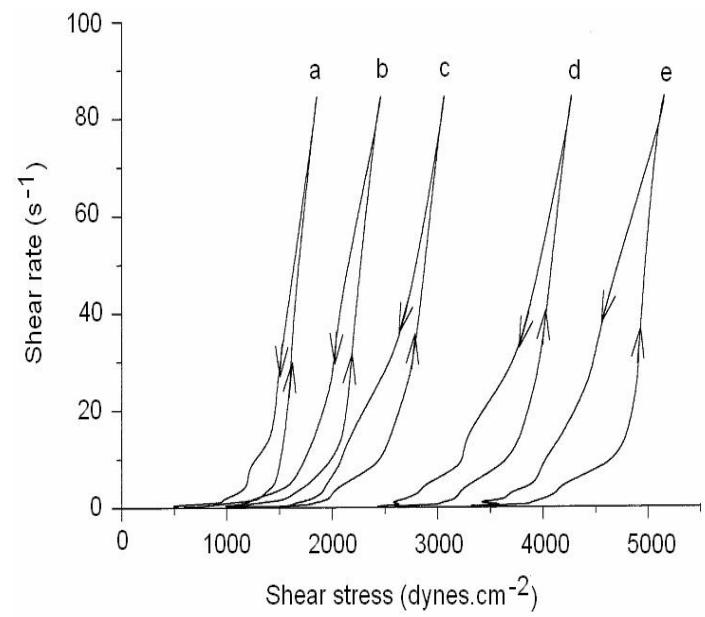

Figure 5: Rheograms of Poloxamer 407 gel $(25 \%$ $\mathrm{w} / \mathrm{v}$ ) at $25^{\circ} \mathrm{C}$ as a function of lecithin concentration: (a) $0 \%$; (b) $1 \%$; (c) $2 \%$; (d) $4 \%$; and (e) $8 \% \mathrm{w} / \mathrm{v}$ [53].

Non-ionic surfactants belonging to the class of poloxamer (Pluronic) and sucrose esters were used both as components of niosomal systems or in the form of sub-micellar solutions. The niosomes obtained were characterized by their entrapment efficiency, size and morphology. The enhancing effect of niosomes on the ex vivo percutaneous penetration of a model drug was investigated using a Franz-type diffusion chamber and compared to that obtained by using submicellar solution of surfactant or achieving pretreatment of the skin with surfactants' submicellar solution or empty niosomes. The results suggest that the surfactants used in this study could be considered as percutaneous permeation enhancers only when they are in the form of drug-loaded vesicular systems. No percutaneous promotion was achieved by using submicellar solution containing free sulfadiazine sodium salt or performing pretreatment with empty niosomes or sub-micellar solutions of the surfactant. Only niosomes were found in their experiments to be effective as transdermal drug delivery systems by Muzzalupo et al [54].

\section{Delivery of big molecules}

Nair and Panchagnula [25] studied the pharmacokinetic and pharmacodynamic activities of arginine vasopressin (AVP), a nanopeptide with antidiuretic activity, on being delivered by transdermal iontophoresis. Poloxamer 407 was used to form stable gels that did not reduce the release of AVP. The release rate from the gel followed Higuchi kinetics indicating that the dominant mechanism of release is diffusion [25]. A matrix gel formulation would be more adaptable from an iontophoretic device point of view. Poloxamer 407 could be used to formulate stable gels of a peptide vasopressin without inhibiting its release from the matrix. The release of peptide from the gel followed Higuchi kinetics indicating that the release was predominantly diffusioncontrolled. The correlation between blood levels and pharmacodynamic activity was only qualitative for vasopressin. Iontophoresis alone and in combination with chemical enhancers which were used to augment the transdermal permeation of AVP. The results of both pharmacokinetic and pharmacodynamic studies emphasize the dimension of 'rapid onset' achieved by iontophoresis. Correlation between pharmacokinetic and pharmacodynamic activities was only 
qualitative. Histopathological studies revealed that skin toxicity, caused by either iontophoresis or chemical enhancers when used alone, could be reduced by using a combination of both techniques in tandem [25].

Insulin has been used as a model peptide for transdermal delivery of large peptides in the molecular weight range of $3-7 \mathrm{kDa}$. A gel formulation of insulin formulated using Poloxamer 407 was evaluated by Pillai and Panchagnula [26] in ex vivo and in vivo skin permeation studies in rat with chemical enhancer and/or iontophoresis. The authors observed that the poloxamer gel was physically and chemically stable during the storage period. In ex vivo studies, both linoleic acid and menthone in combination with iontophoresis showed synergistic enhancement of insulin permeation. Plasma insulin concentration (PIC) was highest with linoleic acid pre-treatment, in agreement with ex vivo permeation studies, but reduction in plasma glucose levels (PGL) was comparable to iontophoresis. Menthone pretreatment resulted in rapid attainment of peak PIC, but reduction in PGL was less than in other treatment groups. They found that there was no direct relationship between PIC and $P G L$, and this was attributed to the fact that the action of insulin is mediated by a cascade of cellular mechanisms, before a reduction in PGL is observed. However, iontophoresis, either alone or in combination with linoleic acid, produced a reduction in $\mathrm{PGL}$ to the extent of $36-40 \%$. A combination of chemical enhancers and iontophoresis caused greater skin irritation than when either of them was used alone [26].

The effect of Poloxamer 188 on electroporation of artificial lipid membranes made of azolectin was investigated by Sharma et al [55]. Two different experimental protocols were used in the study - charge pulse and voltage clamp. Several parameters characterizing the electroporation process were measured and compared with control membranes and membranes treated with 1.0
mM Poloxamer 188. It was found that for both charge pulse and voltage clamp experiments, Poloxamer 188-treated membranes exhibited a statistically higher threshold voltage $(p=$ 0.1 and $p=0.06$, respectively), and longer latency time $(p=0.04$ and $p=0.05$, respectively). These findings suggest that Poloxamer 188 adsorbs into the lipid bilayers, thereby decreasing their susceptibility to electroporation [55].

A Poloxamer 407 gel containing dimethyl isosorbide, isopropyl alcohol, propylene glycol dicaprylocaprate and water has been suggested for permeation enhancement of 5aminolevulinic acid (ALA) across isolated human stratum corneum, in a study by Grüning and Müller-Goymann [56]. A further contribution was the development of a pseudo ternary phase diagram with water, a fixed combination of 1:1 of isopropyl alcohol and dimethyl isosorbide, and a fixed ratio of 4:1 Poloxamer 407 to propylene glycol dicaprylocaprate. The systems were categorized according to their consistencies and ringing gel characteristics with special emphasis on appropriate formulations for dermal application. Polarizing microscopy enabled a clear differentiation between isotropic and anisotropic systems. Wide angle X-ray diffraction analyses confirmed that anisotropy was due to crystalline Poloxamer 407. Furthermore both methods showed that isopropyl alcohol/dimethyl isosorbide was an inferior solvent mixture for Poloxamer 407 related to water [57].

\section{CONLUSION}

One of the developments in technologically optimal vehicles for the administration of drugs is polymeric micelles which improves solubility, stability, bioavailability and biocompatibility depending on the properties of active substance. Long-term investigations indicate that PEO-PPO-based copolymers that display micelle-formation ability provide improvement in solubility and delivery of water insoluble molecules via transdermal way, and they have the advantages of 
protecting drug from first-pass metabolism and providing high patient compliance.

\section{REFERENCES}

1. Chiappetta DA, Sosnik A. Poly(ethylene oxide)poly(propylene oxide) block copolymer micelles as drug delivery agents: Improved hydrosolubility, stability and bioavailability of drugs. Eur J Pharm Biopharm 2007; 66: 303-317.

2. Amidon GL, Lennernas $H$, Shah VP, Crison JR. A theoretical basis for a biopharmaceutic drug classification: the correlation of in vitro drug product dissolution and in vivo bioavailability. Pharm Res 1995; 12: 413-420.

3. Lipinski C. Poor aqueous solubility - an industry wide problem in drug delivery. Am Pharm Rev 2002; 5: 82-85.

4. Kataoka K, Harada A, Nagasaki Y. Block copolymer micelles for drug delivery: design, characterization and biological significance. Adv Drug Del Rev 2001; 47: 113-131.

5. Croy SR, Kwon GS. Polymeric micelles for drug delivery. Curr Pharm Design 2006; 12: 46694684.

6. Moghimi SM, Hunter AC. Poloxamers and poloxamines in nanoparticle engineering and experimental medicine, TIBTECH 2000; 18: 412-420.

7. Aliabadi HM, Lavasanifar A. Polymeric micelles for drug delivery. Exp Opin Drug Del 2006; 3: 139-162.

8. Cafaggi S, Russo E, Caviglioli G, Parodi B, Stefani $R$, Sillo G, Leardi R, Bignardi G. Poloxamer 407 as a solubilising agent for tolfenamic acid and as a base for a gel formulation. Eur $J$ Pharm Sci 2008; 35:19-29.

9. Cafaggi S, Leardi R, Parodi B, Caviglioli G, Bignardi G. An example of application of a mixture design with constraints to a pharmaceutical formulation. Chemom Intell Lab Syst 2003; 65: 139-147.

10. Suh $H$, Jun HW. Physicochemical and release studies of naproxen in poloxamer gels. Int $\mathrm{J}$ Pharm 1996; 129: 13-20.

11. Shin SC, Cho CW, Oh IJ. Enhanced efficacy by percutaneous absorption of piroxicam from the poloxamer gel in rats. Int J Pharm 2000; 193: 213-218.

12. Ye F, Yaghmur A, Jensen H, Larsen SW, Larsen $C$, Østergaard J. Real-time UV imaging of drug diffusion and release from Pluronic F127 hydrogels. Eur J Pharm Sci 2011; 43: 236243.

13. Choi JS, Shin SC. Preparation and Evaluation of Pranoprofen Gel for Percutaneous Administration. Drug Dev Ind Pharm 2007; 33: 19-26.

14. Gonzales N, Sumano $H$. Design of two liquid Ibuprofen-Poloxamer-Limonene or Menthol preparations for dermal administration, Drug Delivery 2007; 14: 287-293.

15. Shin SC, Cho CW, Oh IJ. Effects of non-ionic surfactants as permeation enhancers towards piroxicam from the poloxamer gel through rat skins. Int J Pharm 2001; 222: 199-203.

16. Escobar-Chavez JJ, Quintanar-Guerrero $D$, Ganem-Quintanar A. In Vivo Skin Permeation of Sodium Naproxen Formulated in Pluronic F127 Gels: Effect of Azone and Transcutol. Drug Dev Ind Pharm 2005; 31:447-454.

17. El-Kattan AF, Asbill CS, Kim N, Michniak BB. Effect of Formulation Variables on the Percutaneous Permeation of Ketoprofen from $\mathrm{Gel}$ Formulations. Drug Delivery 2000; 7: 147-153.

18. Lee BJ, Lee TS, Cha BJ, Kim SH, Kim WB. Percutaneous absorption and histopathology of a poloxamer-based formulation of capsaicin analog. Int J Pharm 1997; 159: 105-114.

19. Ur-Rehman T, Tavelin S, Gröbner G. Effect of DMSO on micellization, gelation and drug release profile of Poloxamer 407. Int J Pharm 2010; 394: 92-98.

20. Liaw J, Lin YC. Evaluation of poly(ethylene oxide)poly(propylene oxide)-poly(ethylene oxide) (PEO-PPO-PEO) gels as a release vehicle for percutaneous fentanyl. J Control Release 2000; 68: 273-282.

21. Shin SC, Cho CW, Yang KH. Development of lidocaine gels for enhanced local anesthetic action. Int J Pharm 2004; 287: 73-78.

22. Pandit NK, Wang D. Salt effects on the diffusion and release rate of propranolol from poloxamer 407 gels. Int J Pharm 1998; 167: 183-189.

23. Suedee $R$, Bodhibukkana $C$, Tangthong $N$, Amnuaikit C, Kaewnopparat S, Srichana $T$. Development of a reservoir-type transdermal enantioselective-controlled delivery system for racemic propranolol using a molecularly imprinted polymer composite membrane. J Control Release 2008; 129: 170-178.

24. Stamatialis DF, Rolevink HHM, Koops GH. Transdermal timolol delivery from a Pluronic gel. J Control Release 2006; 116(2): 53-5. doi:10.1016/j.jconrel.2006.09.046

25. Nair V, Panchagnula R. Poloxamer gel as vehicle for transdermal iontophoretic delivery of arginine vasopressin: evaluation of in vivo performance in rats. Pharmacol Res 2003; 47: 555-562.

26. Pillai $O$, Panchagnula $R$. Transdermal delivery of insulin from poloxamer gel: ex vivo and in vivo skin permeation studies in rat using iontophoresis and chemical enhancers. J Control Release 2003; 89: 127-140.

27. Dumortier G, Grossiord JL, Agnely F, Chaumeil JC. A review of poloxamer 407 pharmaceutical and pharmacological characteristics. Pharm Res 2006; 23(12): 2709-28. doi: 10.1007/s11095-006-9104-4.

28. Kabanov AV, Batrakova EV, Alakhov VY. Pluronic block copolymers as novel polymer 
therapeutics for drug and gene delivery. $J$ Control Release 2002; 82(2-3): 189-212.

29. Kabanov AV, Lemieux $P$, Vinogradov S, Alakhov V. Pluronic block copolymers: novel functional molecules for gene therapy. Adv Drug Del Rev 2002; 54: 223-233.

30. Ruel-Garie'py E, Leroux JC. In situ-forming hydrogels-review of temperature-sensitive systems. Eur J Pharm Biopharm 2004; 58: 409-426.

31. Rassing J, Attwood D. Ultrasonic velocity and lightscattering studies on the polyoxyethylenepolyoxypropylene copolymer Pluronic F127 in aqueous solution. Int J Pharm 1983; 13: 4755

32. Zhou Z, Chu B. Anomalous association behavior of an ethylene oxide/propylene oxide $A B A$ block copolymer in water. Macromolecules 1987; 20: 3089-3091.

33. Zhou Z, Chu B. Light-scattering study on the association behavior of triblock polymers of ethylene oxide and propylene oxide in aqueous solution. J Colloid Interface Sci 1988; 126: 171-180.

34. Wanka G, Hoffman $H$, Ulbricht $W$. The aggregation behavior of poly- (oxyethylene) - poly (oxypropylene) - poly - (oxyethylene) - block copolymers in aqueous solution. Colloid Polym. Sci 1990; 268: 101-117.

35. Mortensen K. Block copolymer in aqueous solution: Micelle formation and hard-sphere crystallization. Progr Colloid Polym Sci 1993; 93: 72-75.

36. Schille' $n K$, Glatter O, Brown W. Characterization of a PEO-PPO-PEO block copolymer system, Progr. Colloid Polym. Sci. 93 (1993) 66-71.

37. Bohorquez M, Koch C, Trygstad T, Pandit N. A study of the temperature-dependent micellization of pluronic F127. J Colloid Interface Sci. 1999; 216: 34-40.

38. Cabana A, Ait-Kadi A, Juhasz J. Study of the gelation process of polyethylene oxidepolypropylene oxide-polyethylene oxide copolymer (poloxamer 407) aqueous solutions. J Colloid Interface Sci 1997; 190: 307-312.

39. Mortensen K, Pederson JS. Structural study on the micelle formation of poly(ethylene oxide)poly(propylene oxide)-poly(ethylene oxide) triblock copolymer in aqueous solution. Macromolecules 1993; 26: 805-812.

40. Cao Y, Rodriguez A, Vacanti M, Ibarra C, Arevalo $C$, Vacanti CA. Comparative study of the use of poly(glycolic acid), calcium alginate and pluronics in the engineering of autologous porcine cartilage. J Biomater Sci Polym Ed 1998; 9: 475-487.

41. Barichello JM, Morishita M, Takayama K, Nagai T. Absorption of insulin from Pluronic F-127 gels following subcutaneous administration in rats. Int J Pharm 1999; 184: 189-198.

42. Saim $A B$, Cao $Y$, Weng $Y$, Chang CN, Vacanti MA, Vacanti CA, Eavey RD. Engineering autogenous cartilage in the shape of a helix using an injectable hydrogel scaffold. Laryngoscope 2000; 10: 1694-1697.

43. Scherlund M, Brodin A, Malmsten M. Micellization and gelation in block copolymer systems containing local anesthetics. Int J Pharm 2000; 211: 37-49.

44. Dumortier G, Groissord JL, Agnely F, Chaumeil JC. A review of poloxamer 407 pharmaceutical and pharmacological characteristics. Pharm Res 2006; 23: 2709-2728.

45. Gong CY, Shi S, Dong PW, Yang B, Qi XR, Guo G, Gu YC, Zhao X, Wei YQ, Qian ZY. Biodegradable in situ gel-forming controlled drug delivery system based on thermosensitive PCL-PEG-PCL hydrogel. Part I. Synthesis, characterization, and acute toxicity evaluation. J Pharm Sci 2009; 98: 4684-4694.

46. Reeve L. The poloxamers: their chemistry and medical applications. In: Domb A, Kost Y, Wiseman $D$, editors. Handbook of Biodegradble Polymers, Drug Targeting and Delivery, vol. 7, London, Great Britain: Harwood Academic Publishers; 1997; pp 231249.

47. Chen CC, Fang CL, Al-Suwayeh SA, Leu YL, Fang JY. Transdermal delivery of selegiline from alginate-Pluronic composite thermogels. Int $J$ Pharm 2011; 415: 119-128.

48. Moebus K, Siepmann J, Bodmeier R. Alginatepoloxamer microparticles for controlled drug delivery to mucosal tissue. Eur J Pharm Biopharm 2009; 72: 42-53.

49. Alexandridis $P$, Hatton TA. Poly(ethylene oxide)poly(propylene oxide)-poly(ethylene oxide) block-copolymer surfactants in aqueoussolutions and at interfaces - thermodynamics, structure, dynamics, and modeling. Colloid Surf A Physicochem Eng Aspects 1995; 96: 146.

50. Xiong $X Y$, Tam KC, Gan LH. Polymeric nanostructures for drug delivery applications based on Pluronic copolymer systems. J Nanosci Nanotechnol 2006; 6: 2638-2650.

51. Hsu SH, Leu YL, Hu JW, Fang JY. Physicochemical characterization and drug release of thermosensitive hydrogels composed of a hyaluronic acid/Pluronic F127 graft. Chem Pharm Bull 2009; 57: 453-458.

52. Kim JH, Choi HK. Effect of additives on the crystallization and the permeation of ketoprofen from adhesive matrix. Int $\mathrm{J}$ Pharm 2002; 236: 81-85.

53. Vito 'ria M, Bentley LB, Marchetti JM, Ricardo N, AliAbi Z, Collett JH. Influence of lecithin on some physical chemical properties of poloxamer gels: rheological, microscopic and in vitro permeation studies. Int J Pharm 1999; 193: 49-55.

54. Muzzalupo R, Tavano L, Cassano R, Trombino S, Ferrarelli T, Picci N. A new approach for the evaluation of niosomes as effective 
Yapar \& Inal

transdermal drug delivery systems. Eur $\mathrm{J}$ Pharm Biopharm 2011; 79: 28-35.

55. Sharma V, Stebe K, Murphy JC, Tung L. Poloxamer 188 decreases susceptibility of artificial lipid membranes to electroporation. Biophysical $J$ 1996; 71: 3229-3241.

56. Grüning N, Müller-Goymann CC. Physicochemical characterisation of a novel thermogelling formulation for percutaneous penetration of 5- aminolevulinic acid. J Pharm Sci 2008; 97: 2311-2323.

57. van Hemelrijck C, Müller-Goymann CC. Characterization of a pseudo ternary phase diagram of poloxamer 407 systems for potential application of 5-aminolevulinic acid in photodynamic therapy. Int J Pharm 2011; 420: 297-303. 